\title{
NNLO QCD corrections to dijet production in deep inelastic scattering
}

\section{J. Currie}

Institute for Particle Physics Phenomenology, Department of Physics, University of Durham, Durham, DH1 3LE, UK

E-mail: james.currie@durham.ac.uk

\section{T. Gehrmann}

Department of Physics, University of Zürich, Winterthurerstrasse 190, CH-8057 Zürich,

Switzerland

E-mail: Thomas.Gehrmann@uzh.ch

\section{J. Niehues*}

Department of Physics, University of Zürich, Winterthurerstrasse 190, CH-8057 Zürich,

Switzerland

E-mail: jan@physik.uzh.ch

We present the first computation of two-jet production in deep inelastic scattering at NNLO, fully differential in the kinematic variables. The computation is carried out using the Antenna Subtraction formalism to handle infrared divergences.

Aspects of the subtraction method are discussed. Our results ware compared to a recent analyses by the $\mathrm{H} 1$ collaboration in the high $Q^{2}$ region.

Loops and Legs in Quantum Field Theory

24-29 April 2016

Leipzig, Germany

\footnotetext{
* Speaker.
} 


\section{Introduction}

In this talk we will present the first calculation of the production of two-jet final states in deep inelastic scattering (DIS) to next-to-next-to-leading order accuracy [1]. A large number of dijet events were recorded by the two HERA experiments, H1 [2, 3, 4] and ZEUS [5, 6], at DESY. At leading order in pertubation theory, dijet production is described by the basic scattering processes $\gamma^{*} q \rightarrow q g$ and $\gamma^{*} g \rightarrow q \bar{q}$ [7] and provides a direct measurement of parton distribution functions (PDFs) [8]. Dijet observables are particularly suited for determining the gluon distribution inside the proton in less well constrained regions [9]. The theory uncertainty associated with NLO predictions $[10,11,12]$ is larger than the experimental uncertainty on the measurements and this limits the accuracy of extracting PDFs as well as on the determination of the strong coupling constant $\alpha_{S}$. A NNLO calculation improves the theoretical uncertainty, reducing the discrepancy between experimental and theoretical errors. The QCD amplitudes required for a prediction at NNLO are: the two-loop amplitudes for two-parton final states [14], the one-loop amplitudes for three-parton final states [15] and the tree-level amplitudes for four-parton final states [16]. Real radiations as well as virtual corrections exhibit infrared divergences that have to be handled correctly to enable numerical phase space integration. In our implementation we use the Antenna Subtraction formalism. This subtraction formalism and our results will be discussed in the following sections.

\section{Antenna Subtraction}

A general NNLO cross section can written as follows:

$$
\begin{aligned}
\mathrm{d} \sigma_{\mathrm{LO}} & =\int_{\mathrm{d} \Phi_{m}} \mathrm{~d} \sigma_{\mathrm{B}} \\
\mathrm{d} \sigma_{\mathrm{NLO}} & =\int_{\mathrm{d} \Phi_{m+1}} \mathrm{~d} \sigma_{\mathrm{NLO}}^{\mathrm{R}}+\int_{\mathrm{d} \Phi_{m}} \mathrm{~d} \sigma_{\mathrm{NLO}}^{\mathrm{V}} \\
\mathrm{d} \sigma_{\mathrm{NNLO}} & =\int_{\mathrm{d} \Phi_{m+2}} \mathrm{~d} \sigma_{\mathrm{NNLO}}^{\mathrm{RR}}+\int_{\mathrm{d} \Phi_{m+1}} \mathrm{~d} \sigma_{\mathrm{NNLO}}^{\mathrm{RV}}+\int_{\mathrm{d} \Phi_{m}} \mathrm{~d} \sigma_{\mathrm{NNLO}}^{\mathrm{VV}},
\end{aligned}
$$

where $\mathrm{d} \Phi_{m}$ is phase space measure of the leading order process with $m$ partons in the final state. In dimensionsal regularisation, the virtual corrections contain explicit infrared poles in the dimensional regularization parameter $\varepsilon$, whereas real emissions develop singularities in unresolved momentum configurations. By the KLN theorem these singularities are equal and opposite in sign, such that the sum of corrections at any order is infrared finite. One method to extract and cancel infrared singularities at and across different partonic multiplicities, enabling numerical integration, is Antenna Subtraction [17]. Antenna Subtraction relies thereby extensively on factoristion properties and will be discussed in the following.

In unresolved momentum configurations, colour-order QCD amplitdues factorise into products of universal singular functions and reduced amplitudes, which only depend on the resolved momenta. At NLO, where only a single particle can be unresolved, these singular functions are given by splitting functions and soft eikonal factors in collinear and soft configurations, respectively. This factorisation also holds at NNLO, where up to two particles can be unresolved, requiring additional splitting functions and soft eikonal factors. 
It is also well known that the phase space factorises for appropriate linear, four-momentum conserving mappings. At NLO, this can be expressed for hard radiators $a, b$ and unresolved particle $i$ as

$$
\mathrm{d} \Phi_{m+1}\left(p_{1}, \cdots, p_{a}, p_{i}, p_{b}, \cdots, p_{m+1}\right)=\mathrm{d} \Phi_{m}\left(p_{1}, \cdots, \tilde{p}_{a}, \tilde{p}_{b}, \cdots, p_{m+1}\right) \times \underbrace{\mathrm{d} \Phi_{3}\left(p_{a}, p_{i}, p_{b}\right)}_{\text {antenna phase space }},
$$

and can be extended to NNLO, where up to two particles can become unresolved. Appropriate mappings exist for different initial states and number of partons involved in the map.

In the antenna formalism, subtraction terms are constructed according to singularitiy factorisation:

$$
\mathrm{X}_{m}^{l}\left(p_{a}, p_{2}, \cdots, p_{m-1}, p_{b}\right)\left|\mathbf{M}_{\mathrm{n}}\left(\cdots, \tilde{p}_{a}, \tilde{p}_{b}, \cdots\right)\right|^{2} \mathscr{J}_{n}\left(\cdots, \tilde{p}_{a}, \tilde{p}_{b}, \cdots\right),
$$

where $\mathrm{X}_{m}^{l}$ are the antenna functions containing $l$ loops and $m$ partons. $\mathscr{J}_{n}\left(\cdots, \tilde{p}_{a}, \tilde{p}_{b}, \cdots\right)$ is the jet function and $\mathrm{M}_{\mathrm{n}}$ the reduced matrix element that may contain loops. Antenna functions are exactly equal to the singular functions in unresolved configurations. In general, NNLO subtractions contain antenna functions up to $m=4$ and $l=0$ or $m=3$ and $l=1$. For deep inelastic scattering antenna functions with all partons in the final state (final-final) [17] or only one parton in the intial state (intial-final) [18] were required. The full set of antenna functions contains all colour-ordered infrared QCD singularities up to NNLO. Using the right combination of antenna functions, all singularities in the unresolved phase space regions could be subtracted.

The factorisation of the phase space under the linear momentum maps is used to integrate the antenna function over their antenna phase space

$$
\mathscr{X}_{m}^{l}\left(s_{m}\right)=\frac{1}{|C(\varepsilon)|^{m+l-2}} \int \mathrm{d} \Phi_{\mathrm{X}_{m}^{l}} \mathrm{X}_{m}^{l}\left(p_{1}, \cdots, p_{m}\right) .
$$

with

$$
C(\varepsilon)=\frac{(4 \pi)^{\varepsilon} e^{-\gamma \varepsilon}}{8 \pi^{2}}
$$

to account for coupling constant renormalisation and $s_{m}$ is the invariant mass of the $m$ partons in the antenna. The function on the left hand side of (2.4) contains poles in $\varepsilon$ up to order $2(m+l-2)$. Combining these functions with the mass factorisation kernels from the PDFs, one can define $J_{l}$ operators [24] that match the Catani pole structure

$$
J_{l}\left(p_{a}, p_{b}\right)=I_{l}\left(p_{a}, p_{b}\right)+\text { finite },
$$

where $I_{l}$ is the Catani operator [25].

Integrated subtraction terms are then used to construct part of the subtractions terms at lower partonic multiplicities (added back in with opposite sign), enabling explicit pole cancellation against poles of virtual matrix elements, so that the Antenna Subtraction amounts to adding a "clever" zero overall. For the NNLO correction this amounts to:

$$
\mathrm{d} \sigma_{\mathrm{NNLO}}=\int_{\mathrm{d} \Phi_{m}+2} \overbrace{\left[\mathrm{d} \sigma_{\mathrm{NNLO}}^{\mathrm{RR}}-\mathrm{d} \sigma_{\mathrm{NNLO}}^{\mathrm{S}}\right]}^{\text {IR finite }}+\int_{\mathrm{d} \Phi_{m}+1} \overbrace{\left[\mathrm{d} \sigma_{\mathrm{NNLO}}^{\mathrm{RV}}-\mathrm{d} \sigma_{\mathrm{NNLO}}^{\mathrm{T}}\right]}^{\text {IR finite }}+\int_{\mathrm{d}_{m}} \overbrace{\left[\mathrm{d} \sigma_{\mathrm{NNLO}}^{\mathrm{VV}}-\mathrm{d} \sigma_{\mathrm{NNLO}}^{\mathrm{U}}\right]}^{\text {IR finite }},
$$


where $\mathrm{d} \sigma_{\mathrm{NNLO}}^{\mathrm{S}}, \mathrm{d} \sigma_{\mathrm{NNLO}}^{\mathrm{T}}$ and $\mathrm{d} \sigma_{\mathrm{NNLO}}^{\mathrm{U}}$ are subtraction terms, rendering the double-real, real-virtual and double-virtual corrections infrared finite. Integrated subtraction terms from $\mathrm{d} \sigma_{\mathrm{NNLO}}^{\mathrm{S}}$ are passed to $\mathrm{d} \sigma_{\mathrm{NNLO}}^{\mathrm{T}}$ and $\mathrm{d} \sigma_{\mathrm{NNLO}}^{\mathrm{U}}$, whereas integrated subtraction terms from $\mathrm{d} \sigma_{\mathrm{NNLO}}^{\mathrm{T}}$ are passed to $\mathrm{d} \sigma_{\mathrm{NNLO}}^{\mathrm{U}}$.

The Antenna Subtraction method was implemented into a numerical program (parton-level event generator), into which the jet algorithm used in the experimental measurement as well as any type of event selection cuts were incorporated. A substantial part of the infrastructure of our program is common to other NNLO calculations of jet production observables within the Antenna Subtraction method [19, 20, 21, 22, 23], which are all part of a newly developed code, NNLOJET. To validate our implementation of the tree-level and one-loop matrix elements, we compared the NLO predictions for dijet and trijet production against SHERPA [26] (in DIS kinematics [27]), which uses OpenLoops [28] to automatically generate the one-loop contributions at NLO. The antenna subtraction is then verified by testing the convergence of subtraction terms and matrix elements in all unresolved limits (as documented for example in [29]) and by the infrared pole cancellation between the integrated subtraction terms and the two-loop matrix elements. Furthermore, the stability against varying a small technical cut parameter was verified.

\section{Implementation and results}

As a first application of our calculation, we consider the recent measurement by the $\mathrm{H} 1$ collaboration [4] of dijet production in DIS at high virtuality $Q^{2}$. The measurement was performed on data taken at the DESY HERA electron proton collider at a centre-of-mass energy of $\sqrt{s}=319 \mathrm{GeV}$. Deep inelastic scattering events are selected by requiring the range of lepton scattering variables: exchanged boson virtuality $150 \mathrm{GeV}^{2}<Q^{2}<15000 \mathrm{GeV}^{2}$ and energy transfer in the proton rest system $0.2<y<0.7$. The hadronic final state is boosted to the Breit frame of reference, where the jet clustering is performed using the inclusive hadronic $k_{T}$ algorithm [31] with $E_{T}$ recombination. To ensure that the jets are contained in the calorimeter coverage, a cut on their pseudorapidity is applied in the HERA laboratory frame: $-1.0<\eta_{L}<2.5$. Jets are accepted in the inclusive
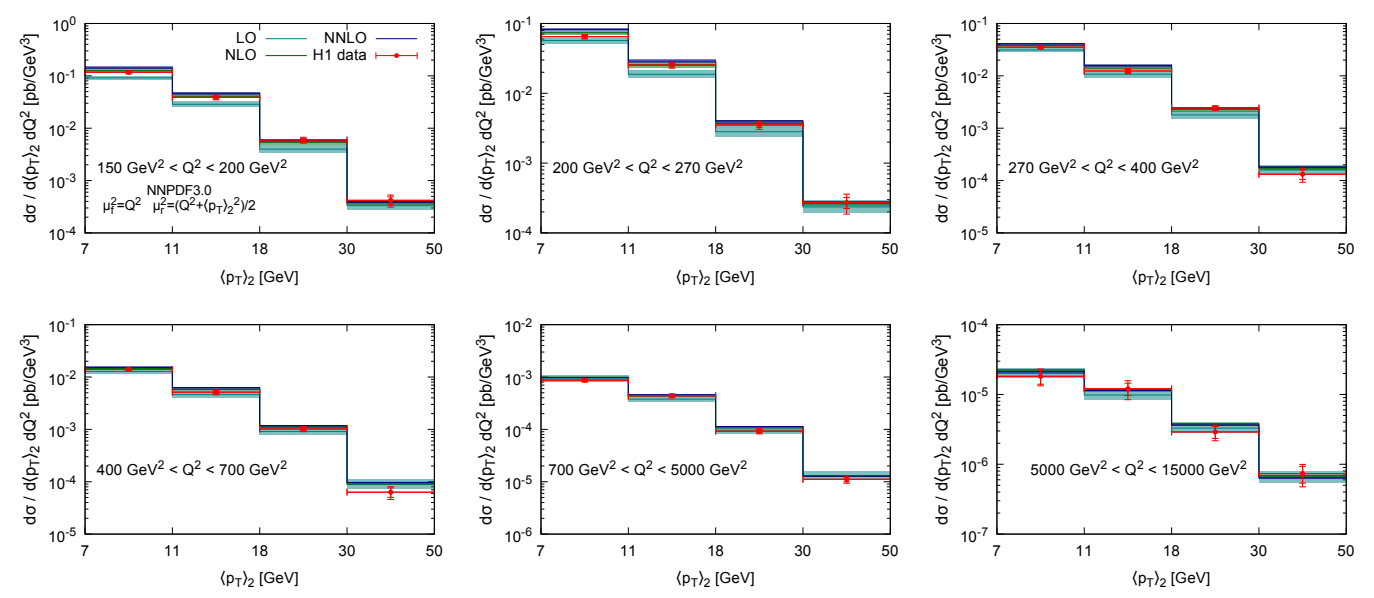

Figure 1: Inclusive dijet production in deep inelastic scattering as function of the average transverse momentum of the two leading jets in the Breit frame at LO, NLO, NNLO, compared to data from the H1 collaboration [4]. 

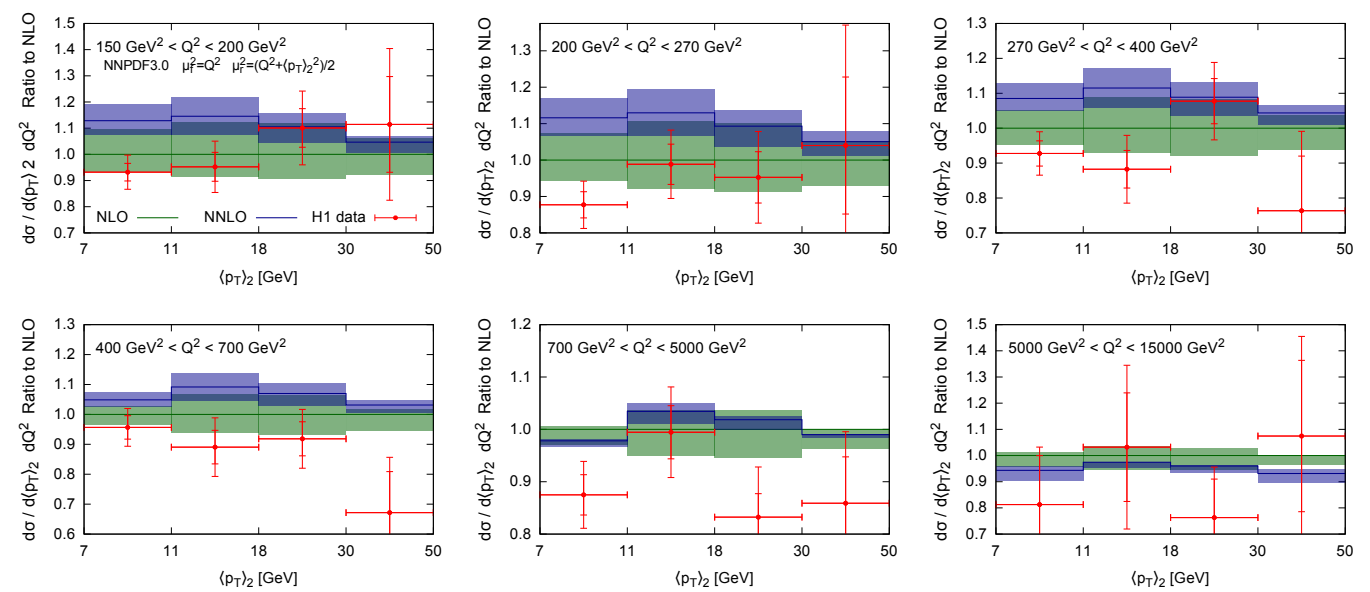

Figure 2: Inclusive dijet production in deep inelastic scattering as function of the average transverse momentum of the two leading jets in the Breit frame normalized to NLO and compared to data from the H1 collaboration [4].

dijet sample if their transverse momentum in the Breit frame is $5 \mathrm{GeV}<p_{T, B}<50 \mathrm{GeV}$ and are ordered in this variable. The event is retained if the invariant mass of the two leading jets is $M_{12}>16 \mathrm{GeV}$. The $\mathrm{H} 1$ collaboration provides double differential distributions in $Q^{2}$ and either the average transverse momentum of the two leading jets $\left\langle p_{T}\right\rangle_{2}=\left(p_{T 1, B}+p_{T 2, B}\right) / 2$ or the variable $\xi_{2}=x\left(1+M_{12} / Q^{2}\right)$ where $x$ is the Bjorken variable reconstructed from the lepton kinematics. At leading order, $\xi_{2}$ can be identified with the proton momentum fraction carried by the parton that initiated the hard scattering process.

The theoretical predictions use the NNPDF3.0 parton distribution functions [13] with $\alpha_{s}\left(M_{Z}^{2}\right)=$ 0.118 and are evaluated with default renormalization and factorization scales $\mu_{F}=\sqrt{Q^{2}}$,

$\mu_{R}=\sqrt{\left(Q^{2}+\left\langle p_{T}\right\rangle_{2}\right)^{2} / 2}$. The uncertainty on the theoretical prediction from missing higher orders is estimated by varying these scales by a factor between $1 / 2$ and 2 . The electromagnetic coupling is also evaluated at a dynamical scale as $\alpha\left(Q^{2}\right)$ according to QED evolution, with $\alpha\left(100 \mathrm{GeV}^{2}\right)=$ 0.0075683 [30]. The theoretical predictions are corrected bin-by-bin for hadronization and electroweak effects using the tables provided in [4].

Figure 1 displays the $\left\langle p_{T}\right\rangle_{2}$ distribution in six $Q^{2}$ bins. For better visibility, the same plots are normalized to the NLO prediction in Figure 2, excluding the LO contribution which is typically considerably below the NLO curve and is associated with a large error. We observe that for all but the first bins in $\left\langle p_{T}\right\rangle_{2}$, the NNLO predictions are inside the NLO uncertainty band and that their inclusion leads to a substantial reduction of the theory uncertainty to typically $5 \%$ or less (especially at high $Q^{2}$ ), which is now below the statistical and systematical uncertainty on the experimental data. We observe that the theoretical NNLO predictions tend to be above the experimental data. This feature points to the potential impact that the inclusion of these data could have in a global determination of parton distributions and of the strong coupling constant at NNLO accuracy. The tension between data and NNLO predictions is largest at lower values of $Q^{2}$, where the data is most accurate and the gluon-induced subprocess dominates the dijet production cross section.

The first bins in $\left\langle p_{T}\right\rangle_{2}$ display a larger correction, often at the upper boundary of the NLO band, 
and only a mild reduction in scale uncertainty. They already have very large NLO corrections, typically with a NLO/LO ratio of about 2 . This feature can be understood from a sophisticated interplay of the $M_{12}>16 \mathrm{GeV}$ cut with the other jet cuts. The $M_{12}$ cut forbids a substantial part of the phase space relevant to the first bin in the $\left\langle p_{T}\right\rangle_{2}$ distribution to be filled by the leading order process. This results in a perturbative instability [32] starting below $\left\langle p_{T}\right\rangle_{2}=8 \mathrm{GeV}$, which leads to a destabilization of the perturbative series for the first bin.

To further illustrate this issue, we display the $\xi_{2}$ distribution in the lowest bin in $Q^{2}$ in Figure 3. The same perturbative instability is present, now spread more uniformly over the first two bins. It is more pronounced than in the $\left\langle p_{T}\right\rangle_{2}$ distribution due to the fact that an even larger fraction of the phase space is forbidden at leading order, since jets down to $p_{T, B}=5 \mathrm{GeV}$ are accepted in this distribution, while maintaining the $M_{12}>16 \mathrm{GeV}$ cut. The resulting instability can already be seen in going from LO to NLO, with substantial corrections outside the nominal scale variation band. In the bins with larger $\xi_{2}$, events with low $M_{12}$ close to the cut are of lower importance, resulting in a better perturbative convergence and a more reliable prediction.

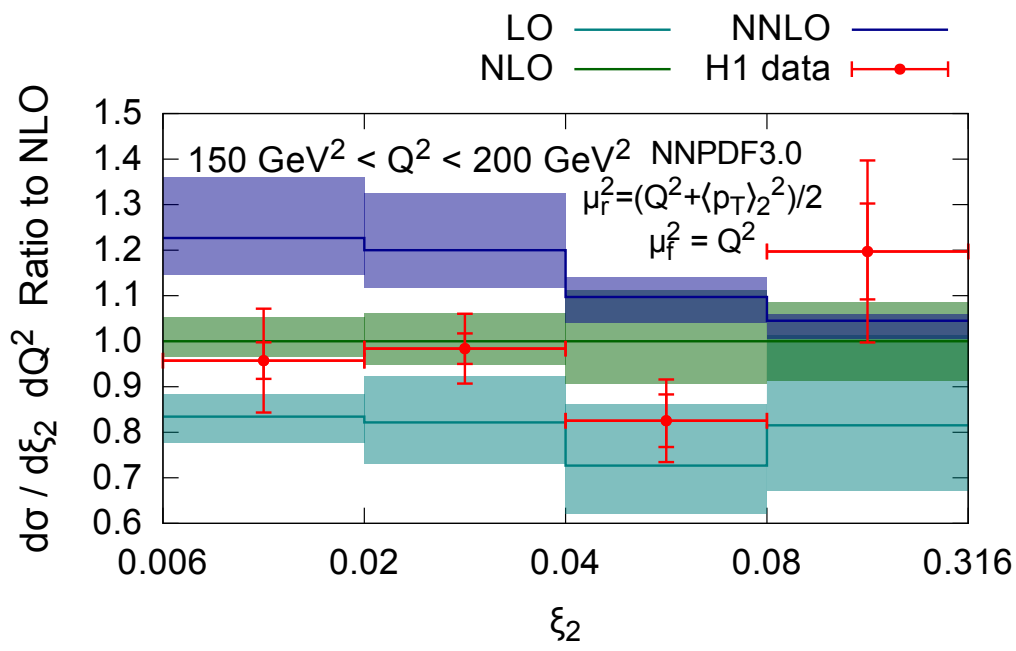

Figure 3: Inclusive dijet production in deep inelastic scattering as function of $\xi_{2}$ normalized to NLO and compared to data from the $\mathrm{H} 1$ collaboration [4].

\section{Conclusions}

In this contribution, we reported on the first calculation of dijet production in deep inelastic scattering to NNLO in QCD using the Antenna Subtraction method. Our results are fully differential in the kinematical variables of the final state lepton and the jets. We applied our calculation to the kinematical situation that is relevant to a recent dijet measurement by the $\mathrm{H} 1$ collaboration [4]. Except for jet production at low transverse momentum (where the experimental event selection cuts destabilize the perturbative convergence), we observe the NNLO corrections to be moderate in size, and overlapping with the scale uncertainty band of the previously available NLO calculation. Especially at lower $Q^{2}$, the NNLO predictions tend to be above the data, which could provide important new information on the gluon distribution at NNLO. The residual uncertainty on the NNLO results 
is of the order of $5 \%$ or less, and below the errors on the experimental data. Our results enable the inclusion of deep inelastic jet data into precision phenomenology studies of the structure of the proton and of the strong coupling constant.

We would like to thank Nigel Glover, Alexander Huss and Thomas Morgan for many interesting discussions throughout the whole course of this project, Stefan Höche and Marek Schönherr for help with the NLO comparisons against SHERPA and Daniel Britzger for useful clarifications on the H1 jet data. This research was supported in part by the Swiss National Science Foundation (SNF) under contract 200020-162487, in part by the UK Science and Technology Facilities Council as well as by the Research Executive Agency (REA) of the European Union under the Grant Agreement PITN-GA-2012-316704 ("HiggsTools"), the ERC Advanced Grant MC@NNLO (340983) and by the National Science Foundation under grant NSF PHY11-25915.

\section{References}

[1] J. Currie, T. Gehrmann and J. Niehues, arXiv:1606.03991 [hep-ph].

[2] A. Aktas et al. [H1 Collaboration], Phys. Lett. B 653 (2007) 134.

[3] F. D. Aaron et al. [H1 Collaboration], Eur. Phys. J. C 67 (2010) 1.

[4] V. Andreev et al. [H1 Collaboration], Eur. Phys. J. C 75 (2015) 65.

[5] S. Chekanov et al. [ZEUS Collaboration], Phys. Lett. B 547 (2002) 164.

[6] H. Abramowicz et al. [ZEUS Collaboration], Eur. Phys. J. C 70 (2010) 965.

[7] K.H. Streng, T.F. Walsh and P.M. Zerwas, Z. Phys. C 2 (1979) 237; R. D. Peccei and R. Rückl, Nucl. Phys. B 162 (1980) 125; C. Rumpf, G. Kramer and J. Willrodt, Z. Phys. C 7 (1981) 337.

[8] R. Devenish and A. Cooper-Sarkar, Deep inelastic scattering, Oxford University Press (Oxford, 2004).

[9] P. Newman, M. Wing, Rev. Mod. Phys. 86 (2014) 1037.

[10] D. Graudenz, Phys. Rev. D 49 (1994) 3291; hep-ph/9710244.

[11] E. Mirkes and D. Zeppenfeld, Phys. Lett. B 380 (1996) 205.

[12] Z. Nagy and Z. Trocsanyi, Phys. Rev. Lett. 87 (2001) 082001.

[13] R. D. Ball et al., JHEP 1504 (2015) 040.

[14] L.W. Garland, T. Gehrmann, E.W.N. Glover, A. Koukoutsakis and E. Remiddi, Nucl. Phys. B 627 (2002) 107; Nucl. Phys. B 642 (2002) 227; T. Gehrmann and E. Remiddi, Nucl. Phys. B 640 (2002) 379; T. Gehrmann and E.W.N. Glover, Phys. Lett. B 676 (2009) 146.

[15] E. W. N. Glover and D. J. Miller, Phys. Lett. B 396 (1997) 257; Z. Bern, L. J. Dixon, D. A. Kosower and S. Weinzierl, Nucl. Phys. B 489 (1997) 3; J. M. Campbell, E. W. N. Glover and D. J. Miller, Phys. Lett. B 409 (1997) 503; Z. Bern, L. J. Dixon and D. A. Kosower, Nucl. Phys. B 513 (1998) 3.

[16] K. Hagiwara and D. Zeppenfeld, Nucl. Phys. B 313 (1989) 560; F. A. Berends, W. T. Giele and H. Kuijf, Nucl. Phys. B 321 (1989) 39; N. K. Falck, D. Graudenz and G. Kramer, Nucl. Phys. B 328 (1989) 317.

[17] A. Gehrmann-De Ridder, T. Gehrmann and E.W.N. Glover, JHEP 0509 (2005) 056; Phys. Lett. B 612 (2005) 49; Phys. Lett. B 612 (2005) 36; J. Currie, E. W. N. Glover and S. Wells, JHEP 1304 (2013) 066. 
[18] A. Daleo, A. Gehrmann-De Ridder, T. Gehrmann and G. Luisoni, JHEP 1001 (2010) 118.

[19] A. Gehrmann-De Ridder, T. Gehrmann, E.W.N. Glover and G. Heinrich, JHEP 0711 (2007) 058; Comput. Phys. Commun. 185 (2014) 3331.

[20] A. Gehrmann-De Ridder, T. Gehrmann, E.W.N. Glover and J. Pires, Phys. Rev. Lett. 110 (2013) 162003;

[21] G. Abelof, A. Gehrmann-De Ridder and I. Majer, JHEP 1512 (2015) 074.

[22] X. Chen, T. Gehrmann, E.W.N. Glover and M. Jaquier, Phys. Lett. B 740 (2015) 147.

[23] A. Gehrmann-De Ridder, T. Gehrmann, E.W.N. Glover, A. Huss and T.A. Morgan, arXiv:1507.02850; arXiv:1605.04295.

[24] J. Currie, A. Gehrmann-De Ridder, E.W.N. Glover and J. Pires, JHEP 1401 (2014) 110.

[25] S. Catani, Phys. Lett. B 427 (1998) 161 S. Catani Phys. Rev. Lett. B427 (1998) 161.

[26] T. Gleisberg, S. Höche, F. Krauss, M. Schönherr, S. Schumann, F. Siegert and J. Winter, JHEP 0902 (2009) 007.

[27] T. Carli, T. Gehrmann and S. Höche, Eur. Phys. J. C 67 (2010) 73.

[28] F. Cascioli, P. Maierhöfer and S. Pozzorini, Phys. Rev. Lett. 108 (2012) 111601.

[29] E.W.N. Glover and J. Pires, JHEP 1006 (2010) 096; A. Gehrmann-De Ridder, E.W.N. Glover and J. Pires, JHEP 1202 (2012) 141.

[30] S. Bodenstein, C. A. Dominguez, K. Schilcher and H. Spiesberger, Phys. Rev. D 86 (2012) 093013

[31] S. Catani, Y.L. Dokshitzer, M.H. Seymour and B.R. Webber, Nucl. Phys. B 406 (1993) 187; G.P. Salam, Eur. Phys. J. C 67 (2010) 637.

[32] S. Catani and B.R. Webber, JHEP 9710 (1997) 005. 